\title{
Objectively measured physical environmental neighbourhood factors are not associated with accelerometer- determined total sedentary time in adults
}

Sofie Compernolle ${ }^{1 *}$ D , Katrien De Cocker ${ }^{1,2}$, Joreintje D. Mackenbach ${ }^{3}$, Femke Van Nassau ${ }^{4}$, Jeroen Lakerveld ${ }^{3}$, Greet Cardon ${ }^{1}$ and IIse De Bourdeaudhuij ${ }^{1}$

\begin{abstract}
Background: The physical neighbourhood environment may influence adults' sedentary behaviour. Yet, most studies examining the association between the physical neighbourhood environment and sedentary behaviour rely on selfreported data of either the physical neighbourhood environment and/or sedentary behaviour. The aim of this study was to investigate the associations between objectively measured physical environmental neighbourhood factors and accelerometer-determined total sedentary time in adults.

Methods: In total, 219 Dutch and 128 Belgian adults (mean age \pm SD: $55.8 \pm 15.4$ years) were recruited between March and August 2014 as part of the European SPOTLIGHT project. Physical environmental neighbourhood factors, grouped into eight domains, i.e. walking, cycling, public transport, aesthetics, land use mix, grocery stores, food outlets and recreational facilities, were assessed using the SPOTLIGHT Virtual Audit Tool. Sedentary time was collected using ActiGraph GT3X+ accelerometers. General linear mixed models were conducted to examine associations between physical environmental neighbourhood factors and total sedentary time.

Results: Participants were sedentary, on average, for $542.9 \mathrm{~min} /$ day (SD: 84.3), or $9.1 \mathrm{~h} /$ day. None of the examined physical environmental neighbourhood factors were significantly related to total sedentary time.

Conclusions: Our findings do not support associations of objectively measured physical environmental neighbourhood factors with adults' objectively sedentary time in Dutch and Belgian adults. More research on sedentary behaviours in settings such as the home and work setting is needed to examine the influence of more specific physical environmental factors on these context-specific sedentary behaviours.
\end{abstract}

Keywords: Sitting time, Built environment, Adults, Neighborhood

\section{Background}

The increase in sedentary behaviour, i.e. any waking activity characterized by an energy expenditure of $\leq 1.5$ metabolic equivalents while in a sitting, reclining or lying position [1], is thought to be an important risk factor for obesity and related chronic diseases, including diabetes mellitus type 2 and cardiovascular diseases [2, 3]. The majority of adults spend too much time sedentary during

\footnotetext{
*Correspondence: sofie.compernolle@ugent.be

'Department of Movement and Sport Sciences, Faculty of Medicine and Health Sciences, Ghent University, Watersportlaan 2, B-9000 Ghent, Belgium Full list of author information is available at the end of the article
}

transport, at work, at home and during leisure $[4,5]$. In order to reduce sedentary time in adults, more insight into the determinants is needed.

Social ecological models describe the potential role of physical environmental factors for sedentary behaviours [6]. These physical environmental factors can be classified into home and institutional environmental factors (e.g. factors in the work environment) and built and natural environmental factors (e.g. factors of the neighbourhood environment) [7]. Physical environmental neighbourhood factors have been recognised as potential determinants of sedentary behaviour by a panel of 
experts in the recently developed Systems of Sedentary behaviours (SOS) framework [7]. For example, the presence of benches in the neighbourhood may directly stimulate residents to be sedentary. Indirectly, the lack of sidewalks and cycle paths in a neighbourhood may increase sedentary time as residents may spend less time on active transportation and more time on passive transportation, or the presence of recreational facilities may encourage residents to go outside, and in this way reduce their sedentary time. Identifying physical environmental neighbourhood factors that are associated with sedentary time is important to inform public health practitioners for developing effective populationbased interventions. Koohsari et al. recently summarized the evidence on associations between neighbourhood environmental factors and sedentary time in a systematic review [8], concluding that there is mixed evidence, if any, for some modest associations. From the 17 included studies in that review, only two studies investigated the association between objectively measured neighbourhood factors and objectively measured total sedentary time $[9,10]$. In the study of Van Dyck et al., examining the association between neighbourhood walkability and sedentary time among 1200 Flemish adults, results showed that residents from high walkable neighbourhoods spent $2.9 \%$ more time sedentary than residents from low walkable neighbourhoods [9]. In the study of Kozo et al., conducted among 2199 adults in the USA, no significant association was observed between neighbourhood walkability and total sedentary time [10]. Of note is that both studies examined the role of walkability. Walkability, defined by residential density, street connectivity and land-use mix, is related to the design of a neighbourhood, and can thus be classified as a macro-scale physical environmental factor. Another study, not included in the review of Koohsari et al., has also recently examined the association between objectively measured macro-environmental neighbourhood factors, such as residential density, intersection density and entertainment density, and objectively measured sedentary behaviour [11]. This study concluded that only net residential density and public transit density were associated with sedentary behaviour [11]. All other studies to date, examined micro-scale environmental factors (i.e. regarding the streetscape) and relied on self-reported data of either the environmental attributes or sedentary time, which may be subject to recall and reporting bias $[8,12]$.

To overcome the limitations of self-reported physical environments and sedentary time, the aim of this study was to examine associations between objectively measured physical environmental neighbourhood factors and accelerometer-determined sedentary time in Dutch and Belgian adults.

\section{Methods}

\section{Study design and sampling}

Data from the present study were derived from the 'Sustainable prevention of obesity through integrated strategies' (SPOTLIGHT) study [13]. In this study, a crosssectional survey was conducted among 6037 adult participants from 60 selected neighbourhoods of five urban regions across Europe: Ghent and suburbs (Belgium), Paris and inner suburbs (France), Budapest and suburbs (Hungary), The Randstad (a conurbation including the cities of Amsterdam, Rotterdam, The Hague, and Utrecht in The Netherlands) and Greater London (UK). Neighbourhoods were defined according to smallscale local administrative boundaries. Sampling of neighbourhoods and recruitment of participants has been described in detail elsewhere [14]. After completing the survey, Dutch and Belgian participants who indicated being interested in future studies, were contacted to participate in an accelerometer study. In total, 255 Dutch and 167 Belgian participants (participation rate $=16 \%$ ) of 24 different neighbourhoods (i.e. 12 Dutch and 12 Belgian neighbourhoods; mean surface is $1.2 \mathrm{~km}^{2}$ and $1.4 \mathrm{~km}^{2}$ respectively) agreed to wear an accelerometer for seven consecutive days. In the Netherlands, the accelerometers were sent to the participants' home addresses including a written instruction on how to wear the device. In Belgium, researchers visited the participants at home to attach the accelerometers and to provide the instructions. In total, informed consents and valid accelerometer data were obtained from 225 Dutch and 149 Belgian participants between March and August 2014. The study was approved by the VU University Medical Centre ethics committee (2012/ 314) and the Ghent University Hospital ethical committee (EC/2013/518).

\section{Measures \\ Accelerometer-determined sedentary time}

The Actigraph triaxial accelerometer (Model GT3X, Actigraph, LLC, Fort Walton beach, FL) was fixed with an elastic belt around the waist on the right side of the participants. Participants were asked to wear the accelerometer for seven consecutive days during waking hours, except during bathing and other water-based activities. A valid day was defined as a day which contained at least $10 \mathrm{~h}$ of accelerometer data, and only adults with at least four valid days were included in the analyses. Each minute of wear-time was classified into sedentary (<100 cpm), light (100-2019 cpm), moderate (2020$5998 \mathrm{cpm})$ and vigorous intensity activity ( $>5999 \mathrm{cpm})$ according to commonly used cut points for adults [15]. Non-wear time was defined as $60 \mathrm{~min}$ of consecutive zeroes, allowing for two interruptions of less than 100 counts per minute [15]. 
Objectively measured physical environmental neighbourhood factors

Objectively measured physical environmental factors of the selected neighbourhoods were assessed using the reliable and valid SPOTLIGHT Virtual Audit Tool (S-VAT) [16]. The S-VAT contains 42 items, grouped into eight domains: walking (six items), cycling (eight items), public transport (two items), aesthetics (nine items), land use mix (three items), grocery stores (five items), food outlets (six items) and recreational facility-related items (three items). All items were assessed at street segment level within the 24 selected neighbourhoods in the Netherlands and in Belgium by trained researchers of the SPOTLIGHT project team. Street segment level data were aggregated to the neighbourhood level by taking the percentage of street segments with each feature in the neighbourhood [17]. Items for which no association with sedentary time was expected based on the SOS framework (e.g. liquor stores, type of residential buildings) [7] were excluded from the statistical analyses, as well as items with limited variance, i.e. if more than half of the neighbourhoods yielded the same percentage (e.g. indoor recreational facilities, take away restaurants, abandoned buildings, and railway/ underground stations). As a result, 16 physical environmental neighbourhood factors were included in the statistical analyses (see Table 2).

\section{Covariates}

Covariates considered in the analyses were retrieved from the online survey, and included age, gender, educational level (lower, higher), and household composition (number of people in the household).

\section{Statistical analysis}

Only participants for whom questionnaire data, S-VAT data and valid accelerometer data were available were included in the current analyses $(N=347)$. Descriptive statistics were computed using $\mathrm{R}$ software, version 3.1.2. to summarize participant characteristics. General linear mixed models were used to examine the associations between physical environmental neighbourhood factors and sedentary time, as the Kolmogorov-Smirnov test revealed a normal distribution of the dependent variable (sedentary time). A random intercept was added within all models to account for clustering of participants at neighbourhood level. Physical environmental neighbourhood items were multiplied by 100 before entering in the models to facilitate interpretation, so that a 1-unit increase represents a $1 \%$ increase in the presence of neighbourhood characteristics.

In a first step, the separate associations between the sixteen selected physical environmental neighbourhood factors and sedentary time were analysed using single regression models. In a second step (model 1), the same physical environmental neighbourhood factors were entered in a multivariable regression model, except the presence of cafés, local food shops, residential gardens, litter, bicycle lanes and public parks. As the variance inflation factors showed that these variables violated the assumption of multicollinearity, these variables were not included in the multivariable regression model. In a final step (model 2), the covariates were added to the model to control for their influence on the potential associations. Statistical significance was set at $p \leq 0.05$.

\section{Results}

\section{Participant characteristics}

Participant characteristics are presented in Table 1. The mean age of the total sample was $55.8(\mathrm{SD}=15.4)$ years, ranging from 19 to 91 years. More than half of the Dutch participants were female (56.7\%), whereas more than half of the Belgian participants were male (54.9\%).

Table 1 Socio-demographic characteristics, body mass index, and sedentary time of the total sample

\begin{tabular}{llll}
\hline & Total $(n=347)$ & Dutch participants $(n=219)$ & Belgian participants $(n=128)$ \\
\hline Age: years, mean (SD) & $55.8(15.4)$ & $57.6(15.1)$ & $52.8(15.5)$ \\
Gender: \% & 47.5 & 43.3 & 54.9 \\
$\quad$ Male & 52.5 & 56.7 & 45.1 \\
$\quad$ Female & & & 15.7 \\
Household composition: \% & 23.2 & 27.6 & 50.4 \\
$\quad$ One-person household & 46.9 & 44.9 & 33.9 \\
$\quad$ Two-person household & 29.9 & 27.5 & 29.3 \\
$\quad$ Three-or more-person household & & & 70.7 \\
Educational level: \% & 36.3 & 40.3 & $518.7(83.4)$ \\
$\quad$ No tertiary education & 63.7 & 56.7 & 556.9 (81.8) \\
$\quad$ Tertiary education & $542.9(84.3)$ & & \\
Total sedentary time: min/day, mean (SD) &
\end{tabular}

$N$ number of participants, $S D$ standard deviation 
Most participants lived in a two-person household (46.9\%), and completed tertiary education. The mean sedentary time was $542.9 \mathrm{~min} /$ day, or $9.05 \mathrm{~h} /$ day.

\section{Physical environmental neighbourhood correlates of accelerometer-determined sedentary time}

Table 2 shows the results of the regression analyses. None of the examined physical environmental neighbourhood factors were significantly associated with accelerometer-determined sedentary time, and only one factor showed a trend towards significance with sedentary time, namely the presence of traffic calming devices $(p=0.06)$. This trend was only visible in the single regression analysis.

\section{Discussion}

This study examined whether a range of objectively measured physical environmental neighbourhood factors were related to adults' accelerometer-determined sedentary time. The results showed that none of the examined physical environmental factors were significantly associated with total sedentary time, suggesting that neighbourhood related factors are not influential for the total amount of time people sit per day. This is in contrast with what was expected from Owen's ecological model of four domains of sedentary behaviour. In this model, the neighbourhood environment is listed as one of the behavioural settings that might influence adults' sedentary time. Essential to note is that in this model, the importance of four different domains of sedentary behaviour (i.e. occupational, household, transport-related and leisure time sedentary time) has also been emphasized, such that specific sedentary behaviours may happen in a variety of behavioural settings [6]. Only two out of four domains of sedentary behaviour might occur partially in the neighbourhood environment, namely

Table 2 Associations between physical environmental neighbourhood factors and total sedentary time $(N=347)$

\begin{tabular}{|c|c|c|c|c|c|c|}
\hline \multirow[t]{2}{*}{$\begin{array}{l}\text { Physical environmental } \\
\text { neighbourhood factors }{ }^{b}\end{array}$} & \multicolumn{2}{|c|}{$\begin{array}{l}\text { Physical environmental factors } \\
\text { separately }(n=347)\end{array}$} & \multicolumn{2}{|c|}{$\begin{array}{l}\text { Model 1: Physical environmental } \\
\text { factors combined }(n=347)\end{array}$} & \multicolumn{2}{|c|}{$\begin{array}{l}\text { Model 2: Adjusted for individual } \\
\text { factors }^{\text {a }}(n=329)\end{array}$} \\
\hline & $\overline{b(S . E .)^{c}}$ & $p$ & $\overline{b(S . E .)^{c}}$ & $p$ & $\overline{b(S . E .)^{c}}$ & $p$ \\
\hline \multicolumn{7}{|c|}{ Presence of food outlets and grocery stores } \\
\hline Restaurants & $0.36(1.39)$ & 0.80 & $1.54(1.66)$ & 0.38 & $1.32(1.74)$ & 0.47 \\
\hline Café/bar & $-0.32(1.59)$ & 0.84 & - & - & - & - \\
\hline Supermarkets & $-4.46(2.71)$ & 0.11 & $-5.77(3.48)$ & 0.14 & $-4.81(3.75)$ & 0.24 \\
\hline Local food shops & $-1.64(2.35)$ & 0.50 & - & - & - & - \\
\hline \multicolumn{7}{|l|}{ Aesthetics } \\
\hline Green/water area & $0.12(0.20)$ & 0.54 & $0.09(0.25)$ & 0.74 & $-0.02(0.27)$ & 0.93 \\
\hline Residential gardens & $0.02(0.19)$ & 0.90 & - & - & - & - \\
\hline Litter & $0.13(0.22)$ & 0.55 & - & - & - & - \\
\hline \multicolumn{7}{|l|}{ Presence of public transport } \\
\hline Presence of bus/tram stops & $-1.93(1.50)$ & 0.21 & $-0.99(2.00)$ & 0.63 & $0.14(2.13)$ & 0.95 \\
\hline \multicolumn{7}{|l|}{ Presence of cycling-related items } \\
\hline Presence of bicycle lanes & $0.87(0.59)$ & 0.16 & - & - & - & - \\
\hline Obstacles on bicycle lanes & $-0.97(0.60)$ & 0.13 & $-0.80(0.62)$ & 0.24 & $-0.75(0.66)$ & 0.30 \\
\hline Traffic calming devices & $0.52(0.26)$ & $0.06^{*}$ & $0.58(0.35)$ & 0.13 & $0.59(0.37)$ & 0.14 \\
\hline \multicolumn{7}{|l|}{ Presence of walking-related items } \\
\hline Pedestrian crossings & $0.43(0.36)$ & 0.25 & $-0.10(0.44)$ & 0.82 & $0.28(0.48)$ & 0.59 \\
\hline Presence of side walks & $-0.20(0.19)$ & 0.30 & $-0.21(0.22)$ & 0.38 & $-0.19(0.24)$ & 0.45 \\
\hline \multicolumn{7}{|l|}{ Land-use mix } \\
\hline Percentage of residential buildings & $0.07(0.74)$ & 0.92 & $0.25(0.84)$ & 0.77 & $0.38(0.88)$ & 0.68 \\
\hline \multicolumn{7}{|l|}{ Presence of physical activity facilities } \\
\hline Outdoor recreational facilities & $2.84(2.84)$ & 0.33 & $-3.19(3.34)$ & 0.37 & $-3.06(3.58)$ & 0.42 \\
\hline Public parks & $-0.72(0.98)$ & 0.47 & - & - & - & - \\
\hline
\end{tabular}

*0.05 $<p<0.10$

ancluded covariates were age, gender, educational level, and household composition

${ }^{b}$ All physical environmental factors are expressed in the percentage of street segments within a neighbourhood including the physical environmental factor 'The b's represent the increase in total sedentary time (minutes/day) with a one unit increase in the physical environmental factors. For example, if there is an increase of $1 \%$ of the street segments within a neighbourhood with outdoor recreational facilities, residents of this neighbourhood will sit 2.84 min more per day 
transport-related sedentary behaviour and leisure-time sedentary behaviour (e.g. sitting in a restaurant). These behaviours might thus directly be influenced by the neighbourhood environment. However, these behaviours generally represent only a small part of total sedentary time $[18,19]$. This may be one explanation for the lack of associations with total sedentary time in our study. The vast majority of adults' sedentary time is attributed to occupational sedentary behaviour and recreational television time $[18,19]$. Occupational sedentary behaviour generally takes place at the workplace and television time generally takes place at home [6]. Consequently, examining the work and home environment will probably be more relevant to reduce total sedentary time than investigating the neighbourhood environment. This has also been argued by the recently developed Systems of Sedentary time (SOS) framework of Chastin et al. [7]. In this framework, research priorities are identified, showing that examining the influence of institutional settings would be the main research focus at present [7].

Besides directly influencing transport-related and leisure-time sedentary behaviour, the neighbourhood might also be indirectly linked to sedentary behaviour. For example, it could have been that attractive neighbourhoods stimulate people to spend time outside, and in this way, reduce people's sitting time at home (e.g. time spend watching television, or using a computer). However, as no associations were found, this hypothesis could not be confirmed.

Furthermore, it should be noted that the included physical environmental neighbourhood factors of the current study were predominantly micro-scale factors related to the neighbourhood streetscape. It might be that macro-scale factors, related to the neighbourhood design, do influence sedentary time in adults as suggested by Van Dyck et al. [9]. However, more research is needed.

The main strengths of this study include the use of the S-VAT to objectively measure physical environmental neighbourhood factors, as well as the use of accelerometers to objectively measure total sedentary time. Using objective measures has the advantage of being unaffected by recall or social desirability bias. The GT3X accelerometers, used in this study, have also an important limitation as they are not able to distinguish between postures, such as sitting and lying or standing still. Nevertheless, Koster et al. showed a high correlation for sedentary time between the GT3X accelerometer and the ActivPAL activity monitor, suggesting that time spent standing is limited [20]. Other limitations of the current study are its cross-sectional design, which does not allow for the establishment of causalities. Secondly, the relatively small study sample recruited from 24 neighbourhoods and consisting of relatively older and urban participants may have limited the power to detect meaningful associations and may limit the generalisability of our results. And finally, the lack of detailed information on objectively measured domain-specific sedentary times hindered us from drawing conclusions on correlates of domain-specific sedentary times. By using global positioning systems or wearable cameras next to accelerometers, determinants of domain-specific sedentary time could be determined objectively in future research [8].

\section{Conclusion}

Our findings do not support associations of objectively measured physical environmental neighbourhood factors with adults' objectively sedentary time in Dutch and Belgian adults. This is an important finding in itself and can inform future studies. Since most sedentary time occurs indoors, more research into the indoor environment at home and at work is recommended.

\section{Acknowledgements}

The authors would like to thank the whole WP3 SPOTLIGHT study group. This study group consists of: Ketevan Glonti, Harry Rutter, Martin McKee (The Centre for Health and Social Change, London School of Hygiene and Tropical Medicine), Helga Bardos, Roza Adany (Department of Preventive Medicine, University of Debrecen), Jean-Michel Oppert, Hélène Charreire, Célina Roda, Thierry Feuillet, Maher Ben-Rebah (Equipe de Recherche en Epidémiologie Nutritionnelle, Université Paris 13), Jeroen Lakerveld, Joreintje Mackenbach (Department of Epidemiology and Biostatistics, EMGO Institute for Health and Care Research, VU Medical Center, Amsterdam), and Ilse De Bourdeaudhuij, Greet Cardon, Sofie Compernolle (Department of Movement and Sport Sciences, Ghent University). The coordinator of the WP3 SPOTLIGHT group is Harry Rutter (Harry.Rutter@lshtm.ac.uk). Coordinator of the SPOTLIGHT project is Jeroen Lakerveld (je.lakerveld@vumc.nl).

\section{Funding}

This work is part of the SPOTLIGHT project funded by the Seventh Framework Programme (CORDISFP7) of the European Commission, HEALTH(FP7-HEALTH2011 -two-stage), Grant agreement No.278186. The content of this article reflects only the authors' views and the European Commission is not liable for any use that may be made of the information contained therein. The funders had no role in study design, data collection and analysis, decision to publish, or preparation of the manuscript.

\section{Availability of data and materials}

The data is not deposited in publicly available repositories due to the rules of the SPOTLIGHT consortium. The data -or parts of the data- are available to be used by others, but under conditions as specified within the SPOTLIGHT data access committee. Restrictions are that the data set contains personal variables that may reveal the identity of participants - e.g. geolocation of house addresses, in addition, there are foreseen analyses that we want to conduct with our data. A SPOTLIGHT data access committee has laid down terms regarding the management, storage, ownership and external access of the data. Contact point: Dr. Jeroen Lakerveld. J.lakerveld@vumc.nl. All authors are affiliated with the SPOTLIGHT project (which we do not refer to as a cohort). Co-authorship is not a pre-requisite for gaining access to the data set.

\section{Authors' contributions}

SC and IDB conceived of the idea for the manuscript. SC conducted the statistical analyses, interpreted the data and drafted the manuscript. SC and JDM conducted the data collection. FVA cleaned the data. All authors read, provided feedback, and approved the final submitted version of the manuscript. 


\section{Ethics approval and consent to participate}

All participants provided informed consent before the start of the study. The study was approved by the corresponding local ethics committees of participating countries. In Belgium, the study was approved by the ethics committee of Ghent University Hospital (EC/2013/518), and in The Netherlands by the Medical Ethics Committee of the VU University Medical Center in Amsterdam (2012/314)

\section{Consent for publication}

Not applicable.

\section{Competing interests}

The authors declare that they have no competing interests.

\section{Publisher's Note}

Springer Nature remains neutral with regard to jurisdictional claims in published maps and institutional affiliations.

\section{Author details}

'Department of Movement and Sport Sciences, Faculty of Medicine and Health Sciences, Ghent University, Watersportlaan 2, B-9000 Ghent, Belgium ${ }^{2}$ Research Foundation Flanders (FWO), Brussels, Belgium. ${ }^{3}$ Department of Epidemiology and Biostatistics, Amsterdam Public Health Research Institute, VU University Medical Center, Amsterdam, The Netherlands. ${ }^{4}$ Department of Public and Occupational Health, Amsterdam Public Health Research, VU University Medical Center, Amsterdam, The Netherlands.

Received: 2 January 2017 Accepted: 7 July 2017

Published online: 14 July 2017

\section{References}

1. Sedentary Behaviour Research Network. Letter to the editor: standardized use of the terms "sedentary" and "sedentary behaviours". Appl Physiol Nutr Metab. 2012;37:540-2.

2. Biswas A, Oh Pl, Faulkner GE, Bajaj RR, Silver MA, Mitchell MS, Alter DA Sedentary time and its association with risk for disease incidence, mortality, and hospitalization in adults: a systematic review and meta-analysis. Ann Intern Med. 2015;162:123-32.

3. Thorp AA, Owen N, Neuhaus M, Dunstan DW. Sedentary behaviors and subsequent health outcomes in adults: a systematic review of longitudinal studies, 1996-2011. Am J Prev Med. 2011;41:207-15.

4. Owen N, Healy GN, Matthews CE, Dunstan DW. Too much sitting: the population health science of sedentary behavior. Exerc Sport Sci Rev. 2010; 38:105-13.

5. Loyen A, Verloigne M, Van Hecke L, Hendriksen I, Lakerveld J, SteeneJohannessen J, Koster A, Donnelly A, Ekelund U, Deforche B, et al. Variation in population levels of sedentary time in European adults according to cross-European studies: a systematic literature review within DEDIPAC. Int J Behav Nutr Phys Act. 2016;13:71.

6. Owen N, Sugiyama T, Eakin EE, Gardiner PA, Tremblay MS, Sallis JF. Adults' sedentary behavior determinants and interventions. Am J Prev Med. 2011; 41:189-96.

7. Chastin SF, De Craemer M, Lien N, Bernaards C, Buck C, Oppert J-M, Nazare J-A, Lakerveld J, O'Donoghue G, Holdsworth M. The SOS-framework (Systems of Sedentary behaviours): an international transdisciplinary consensus framework for the study of determinants, research priorities and policy on sedentary behaviour across the life course: a DEDIPAC-study. Int J Behav Nutr Phys Act. 2016;13:83.

8. Koohsari MJ, Sugiyama T, Sahlqvist S, Mavoa S, Hadgraft N, Owen N. Neighborhood environmental attributes and adults' sedentary behaviors: review and research agenda. Prev Med. 2015;77:141-9.

9. Van Dyck D, Cardon G, Deforche B, Owen N, Sallis JF, De Bourdeaudhuij I. Neighborhood walkability and sedentary time in Belgian adults. Am J Prev Med. 2010;39:25-32.

10. Kozo J, Sallis JF, Conway TL, Kerr J, Cain K, Saelens BE, Frank LD, Owen N. Sedentary behaviors of adults in relation to neighborhood walkability and income. Health Psychol. 2012;31:704.

11. Cerin E, Zhang CJ, Barnett A, Sit CH, Cheung MM, Johnston JM, Lai P-C, Lee RS. Associations of objectively-assessed neighborhood characteristics with older adults' total physical activity and sedentary time in an ultra-dense urban environment: findings from the ALECS study. Health Place. 2016;42:1-10.
12. O'Donoghue G, Perchoux C, Mensah K, Lakerveld J, van der Ploeg H, Bernaards C, Chastin SF, Simon C, O'Gorman D, Nazare J-A. A systematic review of correlates of sedentary behaviour in adults aged 18-65 years: a socio-ecological approach. BMC Public Health. 2016;16:1.

13. Lakerveld J, Brug J, Bot S, Teixeira P, Rutter H, Woodward E, Samdal O, Stockley L, De Bourdeaudhuij I, van Assema P, et al. Sustainable prevention of obesity through integrated strategies: the SPOTLIGHT project's conceptual framework and design. BMC Public Health. 2012;12:793.

14. Lakerveld J, Ben Rebah M, Mackenbach JD, Charreire H, Compernolle S, Glonti K, Bardos H, Rutter H, De Bourdeaudhuij I, Brug J, Oppert J-M. Obesity-related behaviours and BMI in five urban regions across Europe: sampling design and results from the SPOTLIGHT cross-sectional survey. BMJ Open. 2015;5:e008505.

15. Troiano RP, Berrigan D, Dodd KW, Masse LC, Tilert T, McDowell M. Physical activity in the United States measured by accelerometer. Med Sci Sports Exerc. 2008;40:181.

16. Bethlehem JR, Mackenbach JD, Ben-Rebah M, Compernolle S, Glonti K, Bárdos H, Rutter HR, Charreire H, Oppert J-M, Brug J. The SPOTLIGHT virtual audit tool: a valid and reliable tool to assess obesogenic characteristics of the built environment. Int J Health Geogr. 2014;13:52.

17. Feuillet T, Charreire H, Roda C, Ben Rebah M, Mackenbach J, Compernolle S, Glonti K, Bárdos H, Rutter H, De Bourdeaudhuii I. Neighbourhood typology based on virtual audit of environmental obesogenic characteristics. Obes Rev. 2016;17:19-30.

18. Compernolle S, De Cocker K, Roda C, Oppert J-M, Mackenbach JD, Lakerveld J. Glonti K, Bardos H, Rutter H, Cardon G. Physical environmental correlates of domain-specific sedentary Behaviours across five European regions (the SPOTLIGHT project). PLoS One. 2016;11:e0164812.

19. Busschaert C, De Bourdeaudhuij I, Van Cauwenberg J, Cardon G, De Cocker K. Intrapersonal, social-cognitive and physical environmental variables related to context-specific sitting time in adults: a one-year follow-up study. Int J Behav Nutr Phys Act. 2016;13:1.

20. Koster A, Shiroma EJ, Caserotti P, Matthews CE, Chen KY, Glynn NW, Harris TB. Comparison of sedentary estimates between activPAL and hip-and wrist-worn ActiGraph. Med Sci Sports Exerc. 2016:48:1514-22.

\section{Submit your next manuscript to BioMed Central and we will help you at every step:}

- We accept pre-submission inquiries

- Our selector tool helps you to find the most relevant journal

- We provide round the clock customer support

- Convenient online submission

- Thorough peer review

- Inclusion in PubMed and all major indexing services

- Maximum visibility for your research

Submit your manuscript at www.biomedcentral.com/submit
Biomed Central 